\title{
MÁS ALLÁ DE LAPTOPS Y PIZARRAS \\ DIGITALES: LA EXPERIENCIA CHILENA DE INCORPORACIÓN DE TIC EN LA FORMACIÓN INICIAL DE DOCENTES
}

\section{Introducción}

Uno de los principales desafíos para la incorporación pedagógica de las Tecnologías de Información y Comunicación (TIC) en el contexto escolar se relaciona con la manera en que es abordada dentro de la Formación Inicial de Docentes (FID) y en los aprendizajes y experiencias de los futuros profesores, con el fin de conocer, comprender y resignificar el sentido que estos medios tienen para la construcción de ambientes de aprendizaje. Como reflejo de esta necesidad pueden citarse los estudios que revelan el rol clave que juega el docente al momento de decidir la forma de utilizar las TIC en sus prácticas profesionales (Andrews, 2004), los problemas de integración de experiencias formativas con TIC efectivas para los estudiantes de pedagogía (Russell et al., 2003) y lo dificultoso que resultan los procesos de apropiación vivenciado por los docentes en ejercicio (McClintock, 2000, Sancho, 2001).

En atención a este problema, desde 2005 el Ministerio de Educación de Chile desarrolla una política para la inclusión de las TIC en las carreras de pedagogía, mediante la definición de un marco de estándares y competencias (C.E.T., 2006, Silva et al., 2006a, Silva et al., 2006b) y el posterior apoyo al desarrollo de propuestas de innovación que permitan el rediseño curricular y/o formativo para una efectiva integración curricular de estas tecnologías.

En este artículo se discute el objetivo y sentido de estos proyectos, considerando la experiencia internacional, con el fin de caracterizar las estrategias que 18 universidades chilenas están utilizando como parte de la construcción de un itinerario para incorporar a las TIC en el mejoramiento de la FID. 


\section{La experiencia internacional}

Encontrar experiencias sistematizadas de incorporación de las TIC en los currículos de FID no resulta fácil. Esto obliga a explorar diferentes fuentes respecto de lo que se ha venido haciendo internacionalmente. Para lograr esto, se han revisado (i) enfoques propiciados por organismos internacionales, (ii) planes impulsados por agencias públicas nacionales y (iii) acciones de innovación realizadas por algunas universidades.

De su análisis surge una primera estrategia, impulsada por agencias globales (p.e., UNESCO) o iniciativas supranacionales (p.e., la iniciativa europea Ulearn), cuya característica es propiciar redes entre instituciones para facilitar los procesos de seguimiento, evaluación y aprendizaje al incorporar TIC en contextos educativos (ULEARN, 2003, UNESCO, 2002).

En estas iniciativas se propone la existencia de marcos y criterios de referencia que posibiliten la acreditación de calidad de personas o de programas, tal como lo hace la International Society for Technology in Education (ISTE) ${ }^{2}$ o la Office of Superintendent of Public Instruction of Washington State (OSPI) ${ }^{3}$. En ambos casos, resulta clave la existencia de programas de formación y acompañamiento para la mejora y la explicitación de beneficios ante los resultados (HEFCE, 2004, HEFCE, 2006a, HEFCE, 2006b, NCATE, 2000, NCATE, 2006). Particular interés reviste el apoyo directo a personas que actúan como gestores de los programas de formación inicial de docentes, para que lideren innovaciones que contribuyan a la acreditación de carreras o programas, tal como se puede observar en el caso australiano (NSW Institute of Teachers, 2005, NSW Institute of Teachers, 2006).

Otra estrategia interesante es la llevada a cabo en Gran Bretaña, a través del Learning and Skill Council, que promueve la construcción de conocimiento mediante la implementación, seguimiento y transferencia de resultados de experiencias de carácter piloto de un contexto a otro (Learning and Skill Council, 2001).

2 http://www.iste.org/

3 http://www.k12.wa.us/ 
También destaca la sistematización y adecuación de los sistemas de evaluación docente utilizados por las universidades, de manera de sustentar un mecanismo homogéneo, efectivo y eficiente para el mejoramiento continuo de los profesores universitarios, como propicia el Higher Education Funding Council for England ${ }^{4}$.

En el caso de universidades de países desarrollados, una de las estrategias impulsadas es la creación de instancias institucionales de formación para los estudiantes y de soporte para los profesores, que buscan facilitar el uso pedagógico y tecnológico de las TIC. Esto se aprecia en las experiencias de la Melbourne University ${ }^{5}$ y de la Iowa University State 6 . Otras, como la University of Texas at Austin ${ }^{7}$, impulsan el desarrollo de nuevos entornos de aprendizaje universitario, mediante el fortalecimiento de campus virtuales para la educación e-learning.

De igual manera, agencias y universidades implementan iniciativas para el desarrollo e instalación de competencias en los formadores de formadores, constituyendo grupos de trabajo entre pares, quienes, apoyados por expertos, construyen espacios de intercambio y aprendizaje que se aproximan a las Comunidades en Práctica (Wenger, 1998). En el caso de los currículos de formación inicial, se puede citar el caso de las universidades españolas que han incorporado como materia común de estudio, para las carreras de maestría, el curso denominado "Introducción a las Nuevas Tecnologías".

A pesar de esto, se puede afirmar que la incorporación de TIC más allá de la adquisición de destrezas o habilidades tecnológicas aún resulta escasa (Fisher et al., 2006). Como ejemplo de esta preocupación, el Consejo Europeo de Lisboa (2000) señaló la necesidad de adaptar los sistemas de educación y formación a las demandas de la sociedad del conocimiento, subrayando la necesidad de fomentar las nuevas capacidades básicas, en particular aquellas

\footnotetext{
4 http://www.hefce.ac.uk/

5 http://www.unimelb.edu.au/

6 http://www.iastate.edu/

7 http://www.utexas.edu/
} 
relacionadas con las tecnologías de la información, con el objetivo estratégico de convertir a la Unión Europea en una economía basada en el conocimiento al año $2010^{8}$.

Esta preocupación creciente de alinear los contenidos formativos de los futuros profesores para la construcción de una sociedad global y conectada exige resituar la incorporación de la tecnología en las aulas (Delors, 1996). Iluminadora, en este sentido, resulta una de las conclusiones del informe de la European Schoolnet (2005), que afirma: "Se necesita información sobre los programas de formación inicial del profesorado, incluyendo un marco de referencia de las competencias para la formación inicial coherente con la formación continua" (p. 25).

Para alinear a la universidad a estos cambios, es importante reconocer la importancia de la "innovación guiada" (Hannan and Silver, 2005) o proceso planeado, que introduce cambios para una mejora vinculada con el desarrollo social y formativo de la educación superior. Un proceso innovador conlleva intención, planificación y esfuerzo, pero puede fracasar en los resultados. Por ese motivo, la investigación es especialmente importante y relevante, ya que posibilita dos elementos claves: por un lado, dirigir los procesos de innovación a través del conocimiento básico generado por las investigaciones; por otro, evaluar y analizar los resultados de la innovación.

\section{Analizando la estrategia chilena}

La incomunicación entre la política de formación continua de docentes para el uso curricular de las TIC, impulsada por el Ministerio de Educación de Chile, y el énfasis formativo en TIC recogido en los programas de FID que desarrollan las universidades, es un "talón de Aquiles" para la consecución de los objetivos de mejoramiento de los aprendizajes y de inclusión tecnológica propiciados por la política pública.

\footnotetext{
8 Ver conclusiones en http://www.europarl.europa.eu/summits/lisl_es.htm
} 
Por tal motivo, el Centro de Educación y Tecnología (CET ${ }^{9}$ ) ha venido impulsando, desde 2005, un itinerario que busca incorporar a las TIC como un eje temático transversal en la formación de los futuros docentes. Para esto ha desarrollado tres fases de trabajo: (i) la formulación de un marco de referencia que permita orientar un rediseño curricular para la inclusión de las TIC, a través de Estándares TIC para la Formación Inicial de Docentes (Silva et al., 2006a, Silva et al., 2006b), Competencias TIC para el desempeño de docentes en ejercicio y un Mapa de Progreso (K12) destinado a establecer las competencias TIC que deben desarrollar los estudiantes del sistema escolar $^{10}$; (ii) la habilitación de académicos que se desempeñan en carreras de FID y profesionales de la Red de Asistencia Técnica de Enlaces ${ }^{11}$, proveniente de 18 universidades que reciben financiamiento público, para la apropiación del marco de estándares y la formulación de proyectos de carácter piloto, con el propósito de resignificar la inclusión de las TIC en al menos una carrera de FID (Silva et al., 2008), y (iii) el financiamiento y acompañamiento en la implementación de estos proyectos, con el fin de relevar las estrategias exitosas utilizadas, facilitando así su irradiación a otras carreras no sólo de las universidades participantes, sino que del sistema de educación superior en su totalidad.

Analizando lo realizado hasta la fecha, se identifican seis focos alrededor de los cuales los equipos responsables de estos proyectos han centrado su preocupación (ver tabla 1). Esto no quiere decir que los otros focos -o incluso algunos adicionales- no estén presentes; simplemente muestra la importancia que adquiere la existencia de un eje articulador para cada proyecto.

9 Organismo que bajo una nueva institucionalidad impulsa la agenda pública en el campo de la tecnología educativa, a través de la red nacional de universidades que dan forma a la Red Enlaces. Ver http://www.enlaces.cl

10 Estos referentes se estructuran en torno a dimensiones, desde las cuales se desprenden tipos de desempeños que deben ser desarrollados por docentes y estudiantes del sistema escolar. A modo de ejemplo, se puede comentar que el denominado Mapa K12 establece un itinerario de formación para los estudiantes desde NBl a NM4 en cuatro dimensiones: Tecnológica, Información, Comunicación y Ética. Ver http://www.enlaces.cl/competenciastic

11 La RATE está conformada por Centros Zonales y Unidades Ejecutoras conformados en universidades de todo el país. 
Tabla 1. Clasificación de focos de 18 proyectos universitarios

\begin{tabular}{|l|l|}
\hline Foco principal del proyecto & Universidad (de) \\
\hline $\begin{array}{l}\text { Desarrollo de competencias para el trabajo en } \\
\text { entornos virtuales de aprendizaje. }\end{array}$ & Tarapacá, Antofagasta, Santiago de Chile. \\
\hline Mejoramiento del eje de práctica. & $\begin{array}{l}\text { Atacama, Metropolitana de Ciencias de la } \\
\text { Educación, Chile, Magallanes. }\end{array}$ \\
\hline $\begin{array}{l}\text { Fortalecimiento del Perfil de Egreso de } \\
\text { Carreras de Pedagogía. }\end{array}$ & $\begin{array}{l}\text { Católica de Valparaíso, Católica del Maule, } \\
\text { Bío-Bío, La Frontera, Los Lagos. }\end{array}$ \\
\hline Gestión de Conocimiento & La Serena, Playa Ancha. \\
\hline Didácticas Específicas & $\begin{array}{l}\text { Católica de Santiago, Católica de la Santísima } \\
\text { Concepción, Católica de Temuco. }\end{array}$ \\
\hline Centro de Recursos & Concepción. \\
\hline
\end{tabular}

Estos focos son una consecuencia del análisis, contextualización y consenso surgido para responder a la pregunta: ¿cómo superar la visión reduccionista e instrumental que conlleva mirar a las TIC como un conjunto de destrezas operativas? Sus características son las siguientes:

1. Interés por desarrollar competencias en docentes y estudiantes para el uso adecuado de Entornos Virtuales de Aprendizaje (EVA), lo cual resulta un aspecto importante considerando el progresivo aumento de este tipo de espacios, ya sea como complemento a la docencia presencial o como modalidad alternativa de formación. Su eje de atención abarca el desarrollo de habilidades para desenvolverse en estos ambientes y para mediar procesos formativos, en modalidad e-learning o b-learning.

2. Resignificar el aporte de las TIC para el eje de práctica. Bajo este influjo, adquiere relevancia la incorporación de estos medios en la construcción de ambientes de aprendizaje vivenciados de manera real por parte del estudiante en formación en su contacto con los centros de práctica (escuela).

3. Fortalecimiento del perfil del egreso o del marco de competencias de los estudiantes al egresar de la universidad e incorporarse al sistema educativo como profesores novatos. Un elemento clave es la conexión con el proceso de acreditación de la carrera involucrada.

4. Facilitar la gestión de conocimiento por parte de los estudiantes, utilizando herramientas y ambientes sustentados en TIC, mediante procesos formativos centrados en la colaboración entre pares. 
5. Articulación de las TIC con las necesidades de las didácticas específicas, por ejemplo, Lenguaje, Inglés o Ciencias. En estas iniciativas, los esfuerzos de incorporación se centran en conectar las oportunidades ofrecidas por las TIC con las necesidades de los currículos escolares y las estrategias de enseñanza y aprendizaje.

6. Dotar de recursos de aprendizaje a la docencia universitaria, entendiendo que la incorporación de TIC se realiza a través de medios o artefactos que propician un aprendizaje en concordancia con las definiciones y necesidades que las materias y cursos requieren. La creación de Centro de Recursos Digitales para el Aprendizaje es su principal manifestación.

Al analizar las características de estos focos de articulación, resulta posible observar la existencia de tres ejes que interactúan de manera retroactiva como marco referencial para definir la estrategia de incorporación de las TIC (ver figura 1):

Figura 1. Ejes articuladores de las experiencias de innovación chilenas

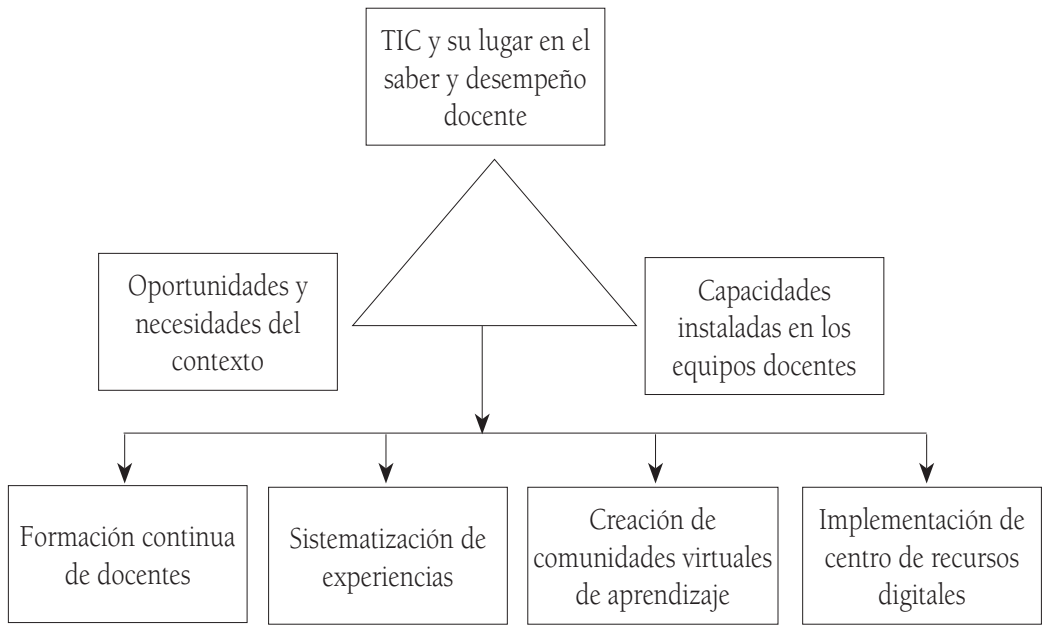

El primero es el análisis de las oportunidades y necesidades propias del contexto, lo que en el caso chileno requiere referirse al factor inductivo que ha jugado el proceso de acreditación, que obliga a las carreras de pedagogía a presentar periódicamente planes 
y compromisos de mejora. Han sido en aquellos contextos que están inmersos o se aproximan a un hito de acreditación donde ha resultado más plausible la conformación de propuestas de inclusión de las TIC.

Un segundo eje presente es la comprensión que cada equipo universitario tiene sobre el rol de las TIC y su impacto sobre el aprendizaje, la formación de los profesores y/o la formación para la vida. Producto de su fundamento epistemológico, conviven perspectivas que las perciben como recursos auxiliares para la enseñanza con aquellas que las resignifican sociocultural y ecosistémicamente dentro del ambiente escolar.

Finalmente el tercer eje tiene relación con las capacidades existentes en docentes y estudiantes, lo que se traduce en una preocupación permanente para que estos actores desarrollen competencias tecnológicas y pedagógicas en el uso de TIC.

Al relacionar comparativamente los focos y ejes surgidos de las 18 experiencias analizadas, emerge una estrategia común conformada por cinco etapas o escalones de complejidad creciente (ver figura 2):

Figura 2. Etapas de la estrategia chilena de incorporación TIC en la FID

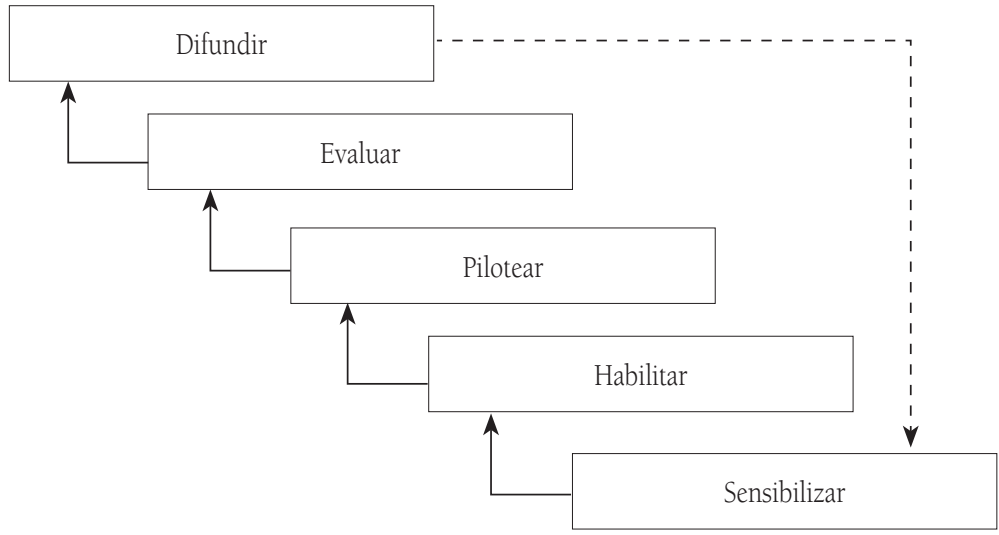

La descripción de cada una de ellas permite caracterizar el camino por el que se ha optado para viabilizar y sustentar las iniciativas de innovación TIC en la FID: 
i. Sensibilización. Etapa inicial imprescindible para el posicionamiento de la innovación en cada universidad. Conlleva, por una parte, la identificación de los actores institucionales claves que deben ser involucrados para realizar la propuesta, lo que en los casos analizados implica indistintamente a vicerrectores, decanos, directores de departamento y/o profesores con reconocimiento o influencia al interior de cada carrera. Por otra parte, y con fuerte retroacción sobre la anterior, reconocer las barreras y oportunidades contextuales que influyen sobre la innovación, por ejemplo la infraestructura, la situación de acreditación o la existencia de asignaturas especializadas.

ii. Habilitación. Segunda etapa destinada al diagnóstico y posterior formación de profesores universitarios en tópicos relevantes para el desarrollo de las propuestas. Destaca el fuerte compromiso por relevar las necesidades realmente sentidas por los participantes, con el fin de definir una oferta de perfeccionamiento y el diseño de propuestas integradoras de aspectos tecnológicos (p.e., uso de software o hardware) y didácticos (p.e., diseño de ambientes de aprendizaje colaborativo).

iii. Pilotear experiencias. El carácter piloto de las innovaciones genera la oportunidad de revisar el lugar de las TIC en el currículo de FID, el que transita desde una perspectiva vertical como "contenido en sí mismo" a una de carácter transversal en el que cada núcleo de formación puede (debe) incorporar el tema. Además de valorar el vínculo entre los propósitos formativos de las carreras de formación inicial y las necesidades contextualmente efectivas de los centros educativos (escuela).

iv. Evaluación. El seguimiento y levantamiento de resultados de las experiencias piloto fortalece la propia perspectiva de incorporación de las TIC, al posibilitar la construcción de un saber pedagógico al interior de cada carrera. En los casos analizados, el principal modo de indagación es el de investigación evaluativa.

v. Difusión. Como último escalón de esta estrategia surge la irradiación de los resultados obtenidos como un medio de legitimación de lo logrado frente a los pares, la que, a su vez, es utilizada como una oportunidad para volver a sensibilizar y desarrollar nuevas propuestas de innovación. 
Este circuito virtuoso de innovación universitaria presenta asimismo aspectos que resulta necesario observar con atención para su mejora, ya que pueden poner en riesgo la vitalidad de la estrategia:

- Sinergia con otros docentes y estudiantes de las carreras donde se implementan las innovaciones, con el fin de ir más allá de los docentes directamente responsables de las iniciativas y de los actores claves de carácter institucional.

- Incorporación de docentes que participan del eje formativo de especialidad o área disciplinaria en torno a la cual gira la carrera de pedagogía. En el caso chileno esto resulta relevante, considerando que los estudiantes se forman en carreras con menciones (p.e., Pedagogía General Básica con mención en Ciencias) o especializaciones (p.e., Pedagogía en Historia o en Matemáticas), que dependen de docentes y muchas veces de departamentos disciplinarios que no están necesariamente integrados con aquellos que se responsabilizan de la formación propiamente pedagógica.

- Funcionamiento de la política de adquisición y uso de recursos TIC, lo que implica dialogar con las instancias responsables de administrar este componente, generalmente de administración centralizada. Ello trae consigo que la petición de una carrera o programa se suma a la totalidad de necesidades existentes en la universidad, retrasando y complejizando la iniciativa.

- Resignificación del marco referencial TIC desde una perspectiva transversal acorde con los Objetivos Fundamentales Transversales (OFT) y el Mapa K12 de desempeños TIC de estudiantes.

- Articulación de las TIC con propósitos curriculares específicos, por ejemplo, sus aportes y límites para la enseñanza o el aprendizaje de Lenguaje, Ciencias o Matemáticas, lo que a su vez surge de la ausencia de investigaciones o resultados claramente diferenciadores del uso o no uso de TIC, lo que dificulta el diálogo y sensibilización académica.

\section{Conclusiones}

Lo que la experiencia chilena muestra es la elaboración de un itinerario que surge de la decisión tomada por un equipo multidisciplinario 
(FID-Red Enlaces) para articular las orientaciones de la política pública con la situación contextual de cada institución.

Esta retroacción muestra que la innovación en el sistema no se produce de manera prescriptiva, sino que es importante diseñar un itinerario y entregar los soportes necesarios para que el profesorado pueda implementar iniciativas de mejoramiento incorporando TIC. De esta manera, las etapas surgidas del análisis a los proyectos entregan pistas para viabilizar este tipo de innovación.

Estas pistas pueden resumirse en tres: (i) mejorar las competencias y capacidades de los equipos docentes que forman profesores, (ii) sistematizar las experiencias con el fin de construir un conocimiento pedagógico que permita fundamentar nuevas innovaciones, procurando construir con ello pequeñas comunidades de aprendizaje entre pares universitarios para el mejor uso de las TIC, e (iii) implementar espacios de innovación para el intercambio de experiencias o el acceso a recursos digitales.

Observado el carácter emergente que poseen estas experiencias, resulta posible identificar algunos desafíos que deberían tenerse en cuenta para profundizar lo realizado:

(a) Impulsar nuevas experiencias que puedan irradiarse más allá de las propuestas piloto realizadas hasta ahora. La cuestión es: ¿de qué manera otras carreras o una facultad completa puede aprender y resignificar el uso de las TIC en la FID?

(b) Propiciar la construcción de conocimiento pedagógico que permita reportar los verdaderos aportes de las TIC a los objetivos de la FID y a las necesidades didácticas y del ejercicio docente que se vivencia en el sistema escolar.

(c) Revisar y actualizar las mallas curriculares para articular competencias TIC provenientes de cada una de sus dimensiones (pedagógica, tecnológica, ética y desarrollo profesional) en los perfiles de egreso de los futuros docentes.

(d) Conectar los objetivos y prácticas realizadas por los docentes formadores de los ejes de práctica, didáctico, metodológico y de especialidad con el marco de estándares y competencias TIC propuesto por el Ministerio de Educación de Chile. 
(e) Propiciar la conformación de redes profesionales con el fin de crear comunidades de práctica, de modo de compartir experiencias, dificultades y soluciones, y recursos didácticos, y planear proyectos colaborativos para abordar la inserción de las TIC en la FID.

Lograr lo anterior permitiría evaluar la penetración y la efectividad (Cuban, 1999) del proceso de cambio llevado a cabo. Para esto, sin embargo, es necesario ampliar y profundizar la investigación sobre la formación y el desempeño del profesorado universitario (formadores) y de los estudiantes (en formación) que interactúan en las aulas o asignaturas de las carreras de FID. Indagar sobre quiénes son, lo que piensan, creen, saben y esperan de este tipo de innovaciones puede ayudar a valorar e incrementar iniciativas de este tipo.

Hacerlo contribuirá al amplio y largo camino que aún resta por recorrer para resituar el fenómeno de las TIC en el eje de formación inicial de docentes, el cual no puede separarse del propio camino que esta transitando la universidad del siglo XXI en su adecuación y posicionamiento en una sociedad global y en red (Bolívar, 2000, Gros, 2006, Perinat, 2004).

\section{Referencias bibliográficas}

Andrews, R. (2004) Conclusion. En Andrews, R. (Ed.). The impact of ICT on literacy education. London: New York, Routledger Falmer.

Bolívar, A. (2000) Globalización y cambio educativo: La sociedad del conocimiento y las claves del cambio. En Estebaranz, A. (Ed.) Construyendo el cambio: Perspectivas y propuestas de innovación educativa. Sevilla: Universidad de Sevilla.

C.E.T. (2006) Estándares en tecnología de la información y la comunicación para la formación inicial docente. Santiago de Chile, Ministerio de Educación.

Cuban, L. (1999) Change without reform in University Curriculo, Teaching, and Research. New York: Teachers College Press.

Delors, J. (1996) La educación encierra un tesoro: Informe UNESCO de la Comisión Internacional sobre educación para el siglo XXI. Madrid: SantillanaUNESCO.

Fisher, T., Higgins, C. \& Loveless, A. (2006) Teachers Learning with Digital Technologies: a review of research and project. Bristol: Future Lab. 
208 MÁS ALLÁ DE LAPTOPS Y PIZARRAS DIGITALES - José Miguel Garrido, Begoña Gros, Jaime Rodríguez, Juan Silva, Hugo Nervi

Gros, B. (2006) La investigación sobre la docencia universitaria: Restos e iniciativas. En Martínez Martin, M. y Carrasco Calvo, S. (eds.) Propuestas para el cambio docente en la Universidad. Barcelona: Octaedro, ICE-UB.

Hannan, A. \& Silver, H. (2005). La innovación en la enseñanza superior. Madrid: Nancea.

HEFCE (2004) Teaching standard: Agenda item, 25 February 2004. London, England: HEFCF.

HEFCE (2006a) Professional Teaching Standards: Publication of Professional Standards Framework 2006, Agenda 26 January 2006, London, England: HEFCF.

HEFCE (2006b). Strategic plan 2006-11. Higher Education Funding Council for England. London, England: HEFCF.

Learning and Skill Council (2001) Quality Improvement Standards Fund 2001/02. Category 1e: Basic Skills Quality Initiative. London: Learning and Skill Council.

McClintock, R. (2000). Prácticas pedagógicas emergentes. Cuadernos de Pedagogía, 74-77.

NCATE (2000) Standards for the accreditation of school media specialist and educational technology specialist programs Bloomington, Association for Educational Communications and Technology.

NCATE (2006) Professional standards accreditation of schools, colleges, and departments of education. Washington D.C.: National Council for Accreditation of Teacher Education.

NSW Institute of Teachers (2005) Professional teaching standards. NSW Institute of Teachers.

NSW Institute of Teachers (2006) Policy and procedures for approval of initial teachers education for programs. Sydney: New South Wales Institute of Teachers.

Perinat, A. (2004) Conocimiento y educación superior: nuevos horizontes para la universidad del siglo XXI. Barcelona: Paidós.

Russell, M., Bebell, D., O’Dwyer, L. \& O'Connor, K. (2003) Examining teacher technology use. Implications for preservice and inservice teacher preparation. Teacher Education, 54, 297-310.

Sancho, J. (2001) Repensando el significado y métodos de la educación en la sociedad de la información: el efecto fractal". En Area, M. (ed.) Educar en la sociedad de la información. Bilbao: Decclée. 
Silva, J., Gros, B., Garrido, J. \& Rodríguez, J. (2006a) Propuesta de estándares TIC para la formación inicial docente. Innovación Educativa, 6, 5-23.

Silva, J., Gros, B., Garrido, J. \& Rodríguez, J. (2006b) Estándares en tecnologías de la información y la comunicación para la formación inicial docente: situación actual y el caso chileno. Revista Iberoamericana de Educación.

Silva, J., Rodríguez, J., Garrido, J., Schalk, A. \& Nervi, H. (2008). Diplomado "Incorporación de Estándares y Competencias TIC en la Formación Inicial Docente". IX Encuentro Internacional Virtual Educa. Zaragoza, España.

ULEARN (2003). ICT in education for pioneer teachers. Italy: European Community.

UNESCO (2002). Information and communication technologies in teacher education. Paris: UNESCO.

Wenger, E. (1998). Communities of practice: Learning, meaning, and identity. Cambridge: Cambridge University Press.

Recibido: 28 de agosto de 2008 Aceptado:25 de septiembre de 2008 